\title{
CORPECTOMIA DA COLUNA TORACOLOMBAR COM COLOCAÇÃO DE CAGE POR ACESSO ÚNICO VIA POSTERIOR: TÉCNICA CIRÚRGICA E RESULTADOS DE SEIS PACIENTES
}

\author{
THORACOLUMBAR SPINE CORPECTOMY WITH PLACEMENT OF A CAGE BY SINGLE \\ POSTERIOR APPROACH: SURGICAL TECHNIQUE AND RESULTS OF SIX PATIENTS \\ CORPECTOMÍA DE LA COLUMNA TORACOLUMBAR CON LA COLOCACIÓN DE CAGE \\ POR ABORDAJE ÚNICO VIIA POSTERIOR: TÉCNICA QUIRÚRGICA Y LOS RESULTADOS \\ DE SEIS PACIENTES
}

Fabiano Morais nogueira ${ }^{1}$, Dionei Freitas de Morais², Rodrigo Antonio Rocha da Cruz Adry ${ }^{3}$, Moysés Isaac Cohen ${ }^{3}$, Renato Andrade Chaves ${ }^{3}$, Gibran Franzoni Rufca ${ }^{3}$, Marco Aurélio Fernandes Teixeira ${ }^{3}$, Sérgio Robinson Martucci Junior ${ }^{3}$

\begin{abstract}
RESUMO
Objetivo: Avaliar retrospectivamente os resultados de uma série de pacientes submetidos à corpectomia torácica e/ou lombar por via exclusivamente posterior associado à colocação de cage e à artrodese instrumentada segmentar e descrever a técnica cirúrgica. Métodos: Foram avaliados retrospectivamente seis pacientes portadores de colapso vertebral, instabilidade biomecânica ou lesão neurológica por diferentes etiologias. Estes pacientes apresentavam indicação de descompressão neural e receberam indicação para a realização de corpectomia e reconstrução circunferencial com cage sendo realizado por via exclusivamente posterior. Resultados: Quatro pacientes eram do sexo masculino e dois do sexo feminino. A idade média foi de 58 anos (22 a 82 anos) com tempo médio de acompanhamento de 10,5 meses (2 a 24 meses). Em três casos a ressecção foi de um corpo vertebral e em três casos de dois corpos vertebrais. Todos os pacientes apresentaram melhora do seu estado neurológico e melhora da dor lombar ou radicular. As indicações ao procedimento foram três casos por espondilodiscite, um por fratura osteoporótica, um tumor metastático e um tumor primário. Três pacientes apresentaram complicações necessitando de revisão cirúrgica evoluindo com melhora dos sintomas. As complicações foram fístula liquórica, radiculopatia lombar, infecção de ferida operatória, meningite e falha da instrumentação. Conclusão: Os pacientes submetidos à corpectomia por via exclusivamente posterior apresentaram resultados favoráveis com melhora do déficit neurológico ou dor em todos os casos. Esta técnica mostrou-se eficiente na reconstrução circunferencial da coluna evitando as complicações da abordagem tradicional por dupla via.
\end{abstract}

Descritores: Coluna vertebral/cirurgia; Fusão vertebral/métodos; Procedimentos ortopédicos/instrumentação; Próteses e implantes; Resultado de tratamento.

\begin{abstract}
Objective: To evaluate retrospectively the results of a series of patients undergone thoracic or lumbar corpectomy via single posterior approach with placement of cage and segmental instrumented arthrodesis. The surgical technique performed was described. Methods: The retrospective study evaluated six patients with vertebral collapse, biomechanical instability or neurological damage caused by different etiologies. These patients showed neural decompression and received indication to perform corpectomy and circumferential reconstruction with cage being conducted exclusively by posterior approach. Results: Four patients were male and 2 female. The average age was 58 years (22-82 years) and mean follow up was 10.5 months (2-24 months). In three cases the resection was only one vertebral body and in three cases two vertebral bodies. All patients improved in neurological status and low back pain or radicular pain. The indications for surgery were three cases of spondylodiscitis, one of osteoporotic fracture, one case of metastatic tumor and one case of primary tumor. Three patients had complications requiring surgical revision; which resulted in improvement of symptoms. The complications that occurred were cerebrospinal fluid leakage, lumbar radiculopathy, wound infection, meningitis and failure of instrumentation. Conclusion: Patients submitted to corpectomy via single posterior approach showed favorable outcomes with improvement of neurological deficit or pain in all cases. This technique was efficient in the reconstruction of circumferential column avoiding the complications of the traditional anterior-posterior approach.
\end{abstract}

Keywords: Spine/surgery; Spinal fusion/methods; Orthopedic procedures/instrumentation; Prostheses and implants; Treatment outcome.

\section{RESUMEN}

Objetivo: Evaluar retrospectivamente los resultados de una serie de pacientes sometidos a corpectomía vertebral por una vía exclusivamente posterior con la colocación de cage y artrodesis instrumentada segmentaria y describir la técnica quirúrgica. Métodos: Estudio retrospectivo evaluó seis pacientes con colapso vertebral, inestabilidad biomecánica y daños neurológicos causados por

1. Neurocirurgião do Serviço de Neurocirurgia da Faculdade Medicina de São José Rio Preto - São José do Rio Preto, Brasil.

2. Professor de Neurocirurgia da Faculdade Medicina de São José Rio Preto - São José do Rio Preto, Brasil.

3. Residentes de Neurocirurgia da Faculdade Medicina de São José Rio Preto - São José do Rio Preto, Brasil.

Trabalho realizado no Serviço de Neurocirurgia da Faculdade de Medicina de São José do Rio Preto.SP.Brasil.

Correspondência: Av. José Munia, 4850.CEP15090-500 - S. J. RioPreto, SP, Brasil. E-mail: fabianorp@ cerebroecoluna.com.br 
diferentes etiologías. Estos pacientes son candidatos para la descompresión neural y recibieron la indicación para la corpectomía y reconstrucción circunferencial con cage por una vía exclusivamente posterior. Resultados: Cuatro pacientes fueron hombres y dos mujeres. La edad promedio fue 58 años (22-82) y el seguimiento medio fue de 10,5 meses (2-24). En tres casos la resección fue un cuerpo vertebral y en tres casos dos vértebras fueron sometidas a resección. Todos los pacientes mejoraron en el estado neurológico, el dolor lumbar o el dolor radicular. Las indicaciones de cirugía fueron tres casos de espondilodiscitis, uno de fractura osteoporótica, un caso de tumor metastásico y un caso de tumor primario. Tres pacientes tuvieron complicaciones que requieren una revisión quirúrgica desarrollada con una mejoría de los síntomas. Las complicaciones fueron pérdida de líquido cefalorraquídeo, la radiculopatía lumbar, infección de la herida, la meningitis y el fracaso de la instrumentación. Conclusión: Los pacientes que se sometieron a corpectomía por una vía exclusivamente posterior mostraron resultados favorables con mejoría del déficit neurológico o dolor en todos los casos. Esta técnica fue eficiente en la reconstrucción de la columna y evitar las complicaciones del doble abordaje.

Descriptores: Columna vertebral/cirugía; Fusión vertebral/métodos; Procedimientos ortopédicos/instrumentación; Prótesis e implantes; Resultado del tratamiento.

\section{INTRODUÇÃO}

A corpectomia é indicada em diversas patologias espinhais que necessitam de descompressão das estruturas neurais associado a reconstrução da coluna anterior. Uma ampla possibilidade de situações clínicas como tumores espinhais primários, metástases, infecções e fraturas envolvendo a integridade do corpo e dos pedículos vertebrais podem implicar na necessidade de se reconstruir a coluna anterior associado a artrodese segmentar instrumentada, conferindo assim maior estabilidade biomecânica ${ }^{1-4}$

Tradicionalmente utiliza-se uma dupla abordagem consistindo em uma via anterior (torácica, toracofrenolombotomia ou lombotomia) para a descompressão das estruturas neurais com colocação do suporte anterior e uma via posterior por incisão mediana para a colocação da instrumentação segmentar. A via combinada pode ser realizada em momentos cirúrgicos distintos ou ainda no mesmo ato anestésico, onde o paciente é reposicionado durante a cirurgia para a realização da dupla abordagem.

O acesso combinado proporciona ampla visualização do corpo vertebral, adequada descompressão das estruturas neurais do canal central e acesso aos elementos posteriores da coluna, porém, ainda que realizada em único tempo cirúrgico como descrita por alguns autores, apresenta importantes limitações relacionadas à necessidade de cirurgião de acesso, maior tempo cirúrgico, maior taxa de sangramento, problemas respiratórias e uma ampla variedade de outras complicações ${ }^{1,5-8}$.

A via posterior como acesso único, realizado em um só tempo cirúrgico, tem sido utilizada por um crescente número de cirurgiões de coluna e tem se mostrado tão eficiente quanto as vias combinadas porém, com menor morbidade, uma vez que as cavidades torácicas e abdominais não são expostas ${ }^{6}$.

O presente artigo descreve os detalhes da técnica cirúrgica utilizada na corpectomia toracolombar associada a colocação de cage em titânio e artrodese instrumentada realizada por via exclusivamente póstero-lateral e apresenta os resultados de seis pacientes submetidos a esta técnica por diferentes indicações clínicas.

\section{CASUÍSTICA E MÉTODOS}

\section{Pacientes e indicações cirúrgicas}

Seis pacientes foram submetidos a corpectomia vertebral na região torácica ou lombar associada a artrodese póstero-lateral instrumentada e reconstrução anterior com cage em titânio por via exclusivamente posterior em um só tempo cirúrgico. Os pacientes foram operados durante o período entre junho de 2008 a junho de 2009 e foram acompanhados clinicamente na Fundação Faculdade Regional de Medicina de São José do Rio Preto e na clínica Centro do Cérebro e Coluna situada na mesma cidade. Destes, quatro eram do sexo masculino e dois do sexo feminino com idade variando de 22 a 82 anos (média de 58 anos). Em três pacientes o processo patológico acometeu duas vertebras sendo indicada a ressecção de ambas (Tabela 1).

As indicações para o procedimento foram variadas incluindo três pacientes com espondilodiscite (sendo um caso confirmado de tuberculose), um caso de metástase por seminoma testicular, um paciente com cordoma acomentendo o corpo vertebral de L4 e um paciente com fratura osteoporótica crônica e dor incapacitante.

Tabela 1. Características pré-operatórias dos pacientes

\begin{tabular}{|c|c|c|c|c|c|}
\hline Caso & \begin{tabular}{|l} 
Idade/ \\
Sexo
\end{tabular} & Diagnóstico & Nível & \begin{tabular}{|c|} 
Status \\
Neurológico \\
pré-operatório \\
(ASIA Scale) \\
\end{tabular} & Procedimento \\
\hline 1 & $82 / F$ & $\begin{array}{c}\text { Fratura } \\
\text { osteoporótica }\end{array}$ & L2 & \begin{tabular}{|c|} 
E \\
(lombalgia \\
incapacitante, \\
não deambula)
\end{tabular} & $\begin{array}{l}\text { Fixação de } \\
\text { T11 a L4; } \\
\text { corpectomia } \\
\text { de L2; sem } \\
\text { amputação } \\
\text { da raiz } \\
\end{array}$ \\
\hline 2 & $66 / F$ & $\begin{array}{l}\text { Espondilodiscite } \\
\text { piogênica } \\
\text { (crônica) }\end{array}$ & $\begin{array}{l}\text { L3 e } \\
\text { L4 }\end{array}$ & \begin{tabular}{|c|}
$\mathrm{E}$ \\
(Iombociatalgia \\
bilateral, não \\
deambula)
\end{tabular} & $\begin{array}{l}\text { Fixação de } \\
\text { T12 a S1; } \\
\text { corpectomia } \\
\text { de L3 e } \\
\text { L4; sem } \\
\text { amputação } \\
\text { de raiz }\end{array}$ \\
\hline 3 & $67 / \mathrm{M}$ & Cordoma & L4 & $\begin{array}{c}E \\
\text { (lombociatalgia } \\
\text { em MIE) }\end{array}$ & $\begin{array}{l}\text { Fixação de } \\
\text { L2 a S1; } \\
\text { corpectomia } \\
\text { de L4; sem } \\
\text { amputação } \\
\text { de raiz }\end{array}$ \\
\hline 4 & $\begin{array}{c}22 / \\
M\end{array}$ & $\begin{array}{l}\text { Metástase } \\
\text { (Seminoma) }\end{array}$ & T10 & B & $\begin{array}{l}\text { Fixação de } \\
\text { T8 a T12; } \\
\text { corpectomia } \\
\text { de T10; com } \\
\text { amputação } \\
\text { raiz T10 a } \\
\text { direita }\end{array}$ \\
\hline 5 & $\begin{array}{l}75 / \\
M\end{array}$ & $\begin{array}{l}\text { Espondilodiscite } \\
\text { tuberculose }\end{array}$ & $\begin{array}{l}\text { T9 e } \\
\text { T10 }\end{array}$ & C & $\begin{array}{c}\text { Fixação de } \\
\text { T6 a T12; } \\
\text { corpectomia } \\
\text { de T9 e T10; } \\
\text { amputação } \\
\text { de raiz T9 a } \\
\text { esquerda }\end{array}$ \\
\hline 6 & $\begin{array}{c}37 / \\
M\end{array}$ & $\begin{array}{l}\text { Espondilodiscite } \\
\text { etiologia } \\
\text { inconclusiva } \\
\text { (crônica) }\end{array}$ & $\begin{array}{l}\text { T12 } \\
\text { e L1 }\end{array}$ & B & $\begin{array}{c}\text { Fixação de } \\
\text { T9 a L3; } \\
\text { corpectomia } \\
\text { de T12 e L1; } \\
\text { amputação } \\
\text { da raiz T12 a } \\
\text { direita }\end{array}$ \\
\hline
\end{tabular}


Todos os pacientes apresentavam colapso do corpo vertebral com instabilidade, ou comprometimento neurológico ou ainda lesão com indicação de ressecção total, na qual o autor entendeu ser necessária a realização da corpectomia com reconstrução circunferencial por via unicamente posterior.

\section{TÉCNICA CIRÚRGICA}

O paciente é posicionado em posição prona sobre coxins que permitem manter o abdome livre de compressões. Realiza-se incisão mediana padrão com dissecção subperiosteal da musculatura dos processos espinhosos com exposição dos elementos posteriores. Na abordagem da coluna torácica é importante expor as costelas aproximadamente $1,5 \mathrm{~cm}$ da articulação costotransversária descolando com uma cureta delicada os tecidos adjacentes subperiosteais ao nível da corpectomia.

Após confirmação do nível da lesão com auxílio fluoroscópico, iniciamos a colocação dos parafusos pediculares seguindo orientações fornecidas pela anatomia e imagens tomográficas. Colocamos a haste apenas de um lado e bloqueamos os parafusos em leve distração para mantermos assim estabilidade e um maior campo cirúrgico durante a corpectomia e colocação do cage. A escolha do lado é contra-lateral ao local de trabalho que por sua vez é determinado pela localização da lesão, acometimento pedicular, quadro clínico do paciente e preferência do cirurgião.

Em seguida realizamos a ressecção do processo espinhoso, laminectomia completa, retirada do ligamento amarelo (flavectomia), dos processos articulares superiores e inferiores (facetectomia) com exposição da duramáter e localização das raízes nervosas. A parte inferior da lâmina logo acima também é ressecada para melhor exposição e descompressão do canal medular. Nesta etapa procede-se a ressecção do processo transverso e da porção proximal da costela onde as articulações costotrasversal e costovertebral são desarticuladas com auxílio do osteótomo.

Em seguida iniciamos a discectomia completa com auxílio de saca-disco, cureta e osteótomo com o cuidado para se evitar escarificação excessiva dos platôs vertebrais, não expondo assim o osso esponjoso das vértebras adjacentes. Antes de se iniciar a corpectomia procedemos a amputação da raíz torácica apenas de um lado afim de se obter maior espaço para a entrada do cage e também evitar a intensa manipulação do saco dural que pode comprometer a integridade medular. Nos casos de corpectomia lombar a amputação da raiz não é realizada pelo risco de déficits motores, sendo realizado leve afastamento da duramáter durante o procedimento de ressecção da vértebra uma vez que ao nível lombar há menor risco de manipulação do saco dural contendo apenas as raízes nervosas.

O aspecto lateral da vértebra é descolado da musculatura paravertebral com gazes, curetas e dissectores tornando a exposição mais ampla, melhorando o ângulo de trabalho na abordagem anterior da vértebra e facilitando a colocação do cage. Neste momento a pleura parietal estará exposta. Em seguida procede-se a retirada dos pedículos com saca-bocados abaixando-os ao nível do corpo vertebral e inicia-se a corpectomia utilizando o drill de alta rotação, curetas grandes, saca-discos fortes e pinças Kerrinson ${ }^{\circledR}$. O aspecto posterior e lateral da vértebra são ressecados por último com o objetivo de proteger a duramáter e os tecidos paravertebrais durante todo o procedimento o qual também é realizado contralateralmente ressecando assim a vértebra por completo.

O cage contendo enxerto ósseo ou metilmetacrilato é introduzido no espaço da vértebra ressecada pelo lado da raiz amputada em posição perpendicular à duramáter (90 graus em relaçao a posiçao final) e em seguida é rodado para posição longitudinal de maneira que permita o suporte aos platôs vertebrais proximal e distal, restabelecendo a coluna anterior (Figura 1).

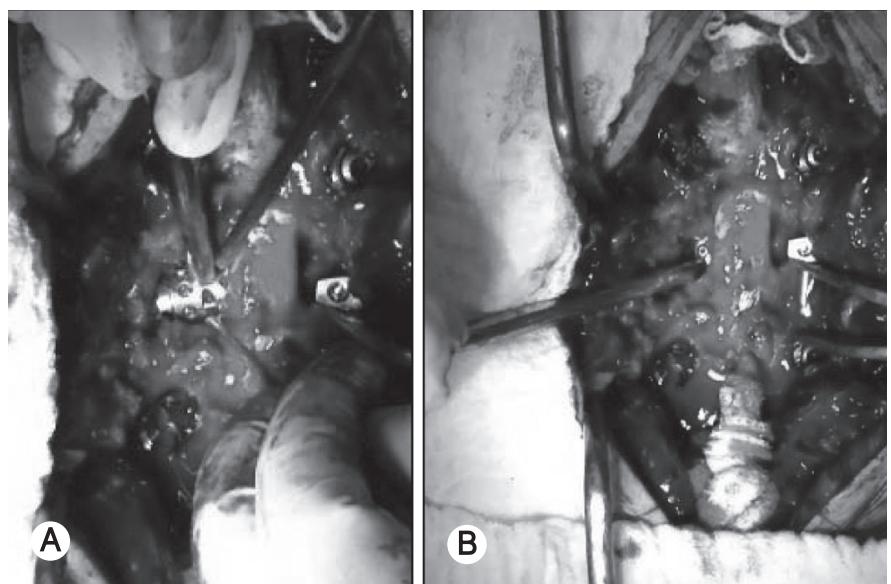

Figura 1. (A) Colocação do cage perpendicular ao saco dural. (B) Giro de 90 graus alinhando o cage longitudinalmente a vertebra proximal e distal.

Dissectores e impactores são utilizados para obter a posiçao final do cage seguido por compressão da instrumentação após confirmado seu correto posicionamento por fluoroscopia. O procedimento é finalizado com o fechamento dos tecidos por planos deixando-se dreno suctor por um a dois dias.

\section{RESULTADOS}

Dentre os seis pacientes operados, três apresentavam-se previamente acamados por déficit neurológico atribuído a compressão medular, apresentando melhora significativa após a descompressão cirúrgica (Tabela 2). Os pacientes quatro e seis apresentavam severo comprometimento medular e foram classificados como ASIA B antes da cirurgia evoluindo para ASIA D em menos de um mês passando a deambular com auxílio apenas do andador. Os outros três pacientes (casos 1, 2 e 3) referiam sintomas de dor lombar mecânica e/ou radicular apresentando melhora clínica evidente após o tratamento cirúrgico, sendo que o caso 3 tornou-se assintomático após a ressecção de cordoma de L4 (Figura 2). O paciente dois foi o único caso a não retornar a deambulação devido a obesidade e hérnia incisional abdominal pós-operatória (tentativa frustrada de corpectomia via retroperitoneal). O paciente evoluiu a óbito por causas clínicas após acompanhamento de 23 meses.

Nenhum paciente necessitou de abordagem anterior, sendo todos realizados por via unicamente posterior. O tempo médio de cirurgia foi de 7,8 horas (variação de 6,5 a 10 horas). Houve três pacientes submetidos a corpectomia de um nível e três pacientes com ressecção de dois níveis totalizando nove vértebras, com instrumentação segmentar posterior de no mínimo dois níveis acima e dois abaixo da vertebrectomia. Foram utilizados dois tipos de cage (Figura 3) de acordo com a disponibilidade no momento da cirurgia, sendo eles o cage cilíndrico ou o cage expansível toracolombar, preenchidos com osso de crista ilíaca ou cimento ósseo. Após a cirurgia todos os pacientes foram submetidos ao Raio-X e/ou tomografia computadorizada os quais apresentaram adequado posicionamento dos implantes (Figura 4) com exceção do paciente cinco que evoluiu com falência da instrumentação e migração do cage para o interior do canal raquimedular.

As complicações relacionadas ao procedimento acometeram três pacientes que necessitaram de revisão cirúrgica havendo resolução em todos os casos. O caso 1 apresentou múltiplas complicações sendo elas: fístula liquórica, radiculopatia de L2 a esquerda, infecção de ferida operatória e meningite. Após revisão cirúrgica e antibioticoterapia houve melhora clínica com cura da infecção e recuperação total das funções da raiz L2. O caso 3 apresentou 
Tabela 2. Características pós-operatórias dos pacientes

\begin{tabular}{|c|c|c|c|c|c|c|}
\hline Caso & $\begin{array}{l}\text { Tipo de } \\
\text { cage }\end{array}$ & $\begin{array}{l}\text { Conteúdo } \\
\text { do cage }\end{array}$ & ASIA pré-op & ASIA pós-op & Complicações & $\begin{array}{c}\text { Seguimento } \\
\text { (meses) }\end{array}$ \\
\hline 2 & $\begin{array}{l}\text { cage } \\
\text { gaiola }\end{array}$ & MMA & $\frac{\mathrm{E}}{\text { (lombociatalgia bilateral) }}$ & $\begin{array}{c}\text { E } \\
\begin{array}{c}\text { (lombalgia mecânica; } \\
\text { não deambula) }\end{array}\end{array}$ & Sem complicações & 23 \\
\hline 4 & $\begin{array}{c}\text { cage } \\
\text { expansível }\end{array}$ & MMA & B & D & Sem complicações & 5 \\
\hline 5 & $\begin{array}{c}\text { cage } \\
\text { expansível }\end{array}$ & MMA & C & D & Falência da instrumentação & 2 \\
\hline 6 & $\begin{array}{c}\text { cage } \\
\text { expansível }\end{array}$ & $\begin{array}{c}\text { osso } \\
\text { autólogo }\end{array}$ & B & D & Sem complicações & 2 \\
\hline
\end{tabular}

MMA: metilmetacrilato
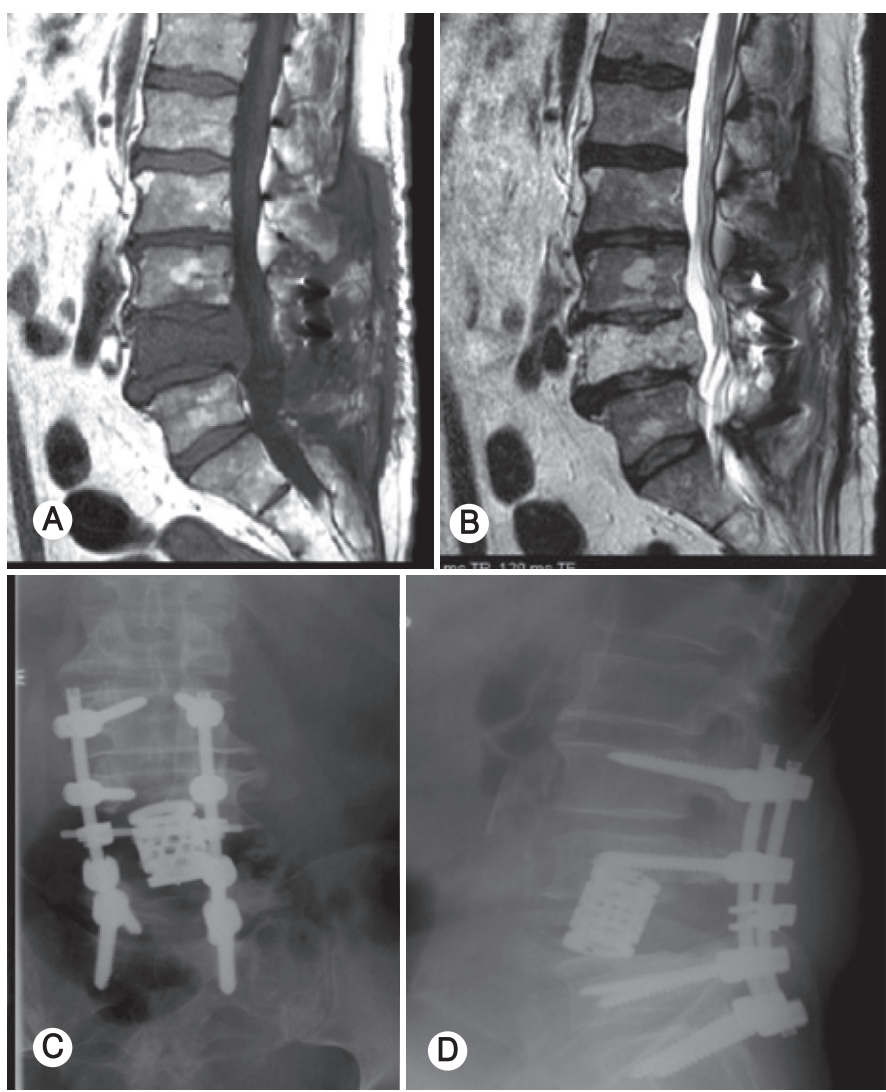

Figura 2. (Cordoma de L4 (caso 3). Ressonância magnética antes do procedimento de corpectomia: (A) corte sagital em T1; corte sagital em T2 (B). Raio-x pós operatório: (C) em AP; perfil (D).

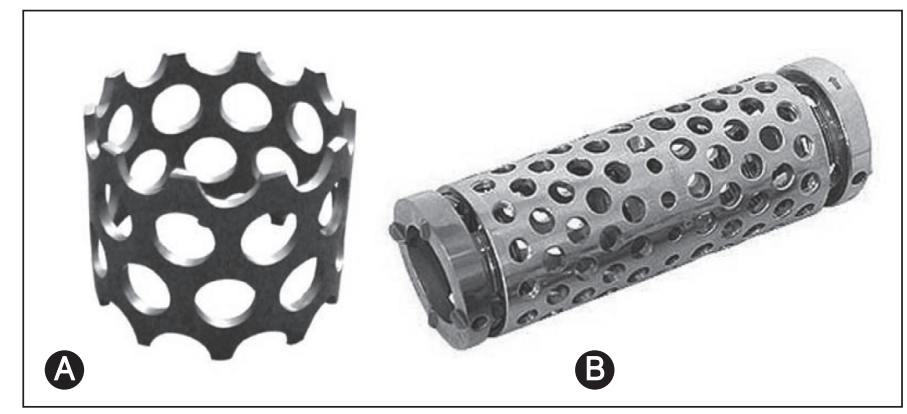

Figura 3. (A) Cage cilíndrico. (B) Cage expansível toracolombar.

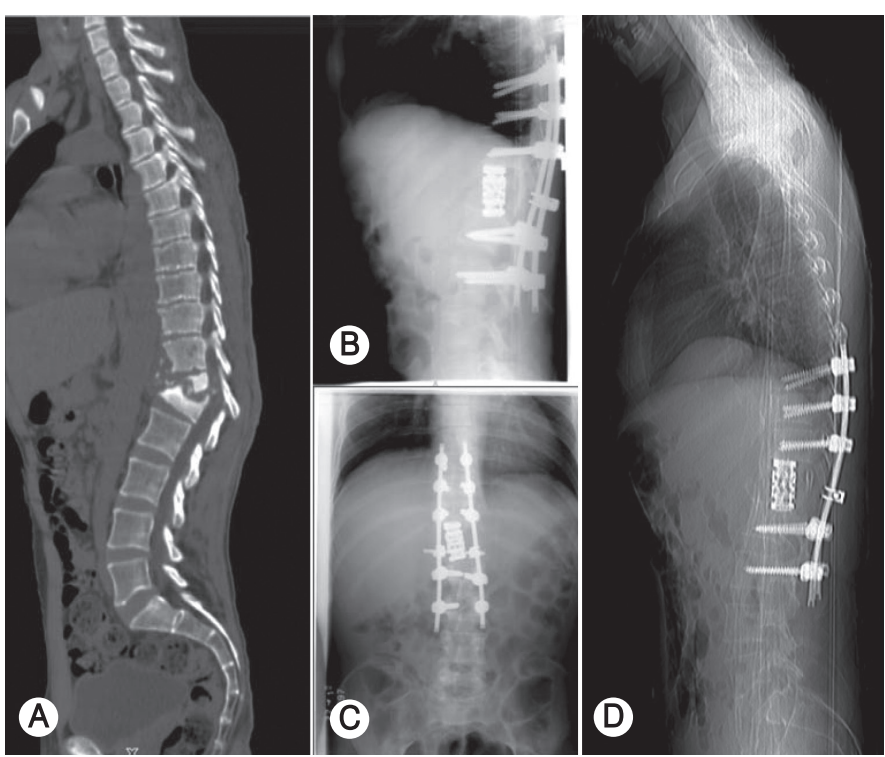

Figura 4. Espondilodiscite crônica (caso 6). Tomografia computadorizada (TC) pré-operatória de coluna toracolombar (A). Raio-X pós-operatório: perfil (B); AP (C). Scanograma de TC da coluna toracolombar (D).

fístula liquórica corrigida cirurgicamente após sete dias do procedimento inicial com resolução do caso. Finalmente o caso 5 apresentou falha da instrumentação após três semanas da cirurgia sendo necessário reposicionamento do cage e aumento distal do nível de instrumentação, evoluindo com melhora da dor e retorno ao estado neurológico inicial.

O acompanhamento clínico após a cirurgia variou de dois meses a dois anos e somente um paciente evoluiu a óbito neste período. Os pacientes submetidos a cirurgia por tumor primário, tumor metastático ou infecção não apresentaram recidiva da doença até o presente momento.

\section{DISCUSSÃO}

As diversas patologias que acometem o corpo vertebral associadas a compressão dos elementos neurais são desafiantes para o cirurgião de coluna que encontra-se na posição de definir a indicação cirúrgica e sua via de acesso.

Nestes casos, a tradicional via anterior (toracotomia ou retro/ transperitoneal) é realizada para a descompressão do saco dural 
e reconstrução da coluna anterior seguida por uma segunda abordagem por via posterior para a instrumentação segmentar. A esta dupla via foram atribuídos alguns benefícios como por exemplo a ampla descompressão neural e a melhora dos déficits sensitivo-motores porém acompanhada de incovenientes relacionados a necessidade de cirurgião de acesso, cirurgia em dois tempos e maior morbidade atribuída principalmente a toracotomia como pneumonia, insuficiência respiratória e lesão vascular ${ }^{8}$ A média de idade dos pacientes que necessitam deste procedimento geralmente ultrapassa os 50 anos ${ }^{5,9}$ e comorbidades clínicas podem ser fatores limitantes a múltiplos procedimentos tornando a via exclusivamente posterior mais atraente.

A experiência inicial em nosso serviço na realização da corpectomia por via posterior exclusiva em seis pacientes tem demonstrado resultados clínicos favoráveis onde foi possível obter a melhora do estado neurológico (Figura 5) e dos sintomas álgicos. Shen et $\mathrm{al}^{10}$ descreveu uma série de 21 casos com compressão medular por tumor vertebral submetidos a corpectomia por via posterior onde todos apresentaram melhora ou mantiveram o seu estado neurológico prévio, com exceção de apenas um caso com deterioração neurológica após tração da raiz nervosa lombar. Em nossa casuística a paciente referente ao caso 1 manifestou déficit transitório da raiz de $\mathrm{L} 2$ a esquerda provavelmente devido a excesso de distração, porém evoluiu com melhora completa após três meses do pós-operatório. A distração da instrumentação a fim de facilitar a entrada do cage deve ser realizada com cautela devido o risco de dano medular ou radicular.

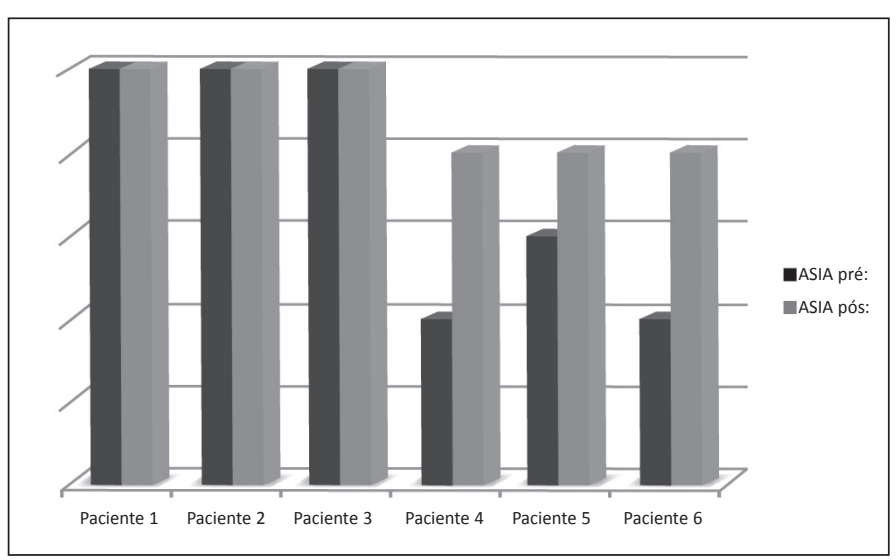

Figura 5. Escala de ASIA pré e pós-operatória.

\section{REFERÊNCIAS:}

1. Hu Y, Xia Q, Ji J, Miao J. One-stage combined posterior and anterior approaches for excising thoracolumbar and lumbar tumors: surgical and oncological outcomes. Spine (Phila Pa 1976). 2010;35(5):590-5.

2. Yoo C, Ryu SI, Park J. Fracture-related thoracic kyphotic deformity correction by singlestage posterolateral vertebrectomy with circumferential reconstruction and stabilization: Outcomes in 30 cases. J Spinal Disord Tech. 2009:22(7):492-501.

3. Crocker M, James G, Ibrahim A, Thomas N, Chitnavis B. Posterior approach bvertebrectomy in the thoracolumbar spine with expandable cage reconstruction: indications and techniques based on eight cases. Br J Neurosurg. 2008;22(2):235-40.

4. Disch AC, Schaser KD, Melcher I, Luzzati A, Feraboli F, SchmoelzW. En bloc spondylectomy reconstructions in a biomechanical in-vitro study. Eur Spine J. 2008;17(5):715-25.

5. Snell BE, Nasr FF, Wolfla CE. Single-stage thoracolumbar vertebrectomy with circumferential reconstruction and arthrodesis: surgical technique and results in 15 patients. Neurosurgery. 2006;58(4)Suppl 2:ONS-263-8.

6. Street J, Fisher C, Sparkes J, Boyd M, Kwon B, Paquette S, et al. Single-stage posterolateral vertebrectomy for the management of metastatic disease of the thoracic and lumbar spine: a prospective study of an evolvingn surgical technique. J Spinal Disord Tech. 2007;20(7):509-20.

7. Fourney DR, Abi-Said D, Rhines LD, Walsh GL, Lang FF, McCutcheon IE et al. Simultaneous anterior-posterior approach to the thoracic and lumbar spine for the radical resection of tumors followed by reconstruction and stabilization. J Neurosurg. 2001;94(2 Suppl):232-44.

8. Sundaresan N, Steinberger AA, Moore F, Sachdev VP, Krol G, Hough L, et al. Indications
A colocação do cage requer cuidado especial com a duramáter que deve ser gentilmente retraída em conjunto com a raiz nervosa permitindo espaço suficiente e seguro para sua entrada. As durotomias não intencionais podem ocorrer neste momento causando fístulas liquóricas muitas vezes na parte anterior do saco dural oferecendo maior dificuldade no seu reparo e detecção. Dois pacientes do estudo apresentaram fístula liquórica que necessitaram revisão cirúrgica. A técnica para a colocação do cage expansível descrita por alguns autores recomenda a entrada do cage pelo ombro da raiz em ângulo de $90^{\circ} \mathrm{com}$ o saco dural e em seguida rotacionado para a posição final com auxílio de dissectores podendo estar o cage amarrado por fitas afim de facilitar sua manipulação ${ }^{11,12}$. Colocamos cage expansível em quatro pacientes, porém o modelo utilizado não permite sua expansão in-situ necessitando de uma chave rosqueada ao cage para manobrá-lo en loco até sua posição final.

O uso do metil metacrilato (MMA) no interior do cage foi utilizado em quatro pacientes evitando assim a retirada de enxerto óssea da crista ilíaca, local frequente de queixas álgicas, permitindo assim a reconstrução da coluna anterior com eficiente estabilização biomecânica da coluna ${ }^{13}$. Os pacientes selecionados para uso do MMA apresentavam como principais diagnósticos tumor vertebral e espondilodiscite, sendo neste último caso realizado desbridamento e instrumentação apenas nos casos crônicos sem melhora ou apresentando sintomas neurológicos ${ }^{14}$

As taxas de complicação variam de 11 a 48\% ${ }^{15}$ mantendo-se inferiores as complicações da dupla via ${ }^{16}$. Embora metade dos pacientes em estudo tenha apresentado complicações acreditamos que a pequena amostra e a fase inicial da curva de aprendizado tenha contribuído para este dado. Nenhum paciente apresentou comprometimento do resultado final após as complicações que foram solucionadas com revisão cirúrgica. As principais foram fístula liquórica, radiculopatia e falência da instrumentação. Assim como outros autores que preconizam esta técnica não encontramos complicações relacionadas a amputação da raiz torácica não havendo queixas de neuralgia intercostal até o momento².

\section{CONCLUSÃO}

Os pacientes submetidos a corpectomia torácica ou lombar por via exclusivamente posterior associado a artrodese instrumentada segmentar, apresentaram resultados favoráveis com melhora do déficit neurológico e da dor em todos os casos. Esta técnica mostrou-se eficiente na reconstrução circunferencial da coluna apresentando baixa morbidade e evitando as complicações inerentes a abordagem tradicional por dupla via.

and results of combined anterior-posterior approaches for spine tumor surgery. J Neurosurg. 1996;85(3):438-46.

9. Cahill DW, Kumar R. Palliative subtotal vertebrectomy with anterior and posterior reconstruction via a single posterior approach. J Neurosurg. 1999; 90(1 Suppl):42-7.

10. Shen FH, Marks I, Shaffrey C, Ouellet J, Arlet V. The use of an expandable cage for corpectomy reconstruction of vertebral body tumors through a posterior extracavitary approach: a multicenter consecutive case series of prospectively followed patients. Spine J. 2008:8(2):329-39.

11. Hunt T, Shen FH, Arlet V. Expandable cage placement via a posterolateral approach in lumbar spine reconstructions. Technical note. J Neurosurg Spine. 2006;5(3):271-4.

12. Morales Alba NA. Posterior placement of an expandable cage for lumbar vertebral body replacement in oncologic surgery by posterior simple approach: technical note. Spine (Phila Pa 1976). 2008;33(23):E901-5

13. Shannon FJ, DiResta GR, Ottaviano D, Castro A, Healey JH, Boland PJ. Biomechanical analysis of anterior poly-methyl-methacrylate reconstruction following total spondylectomy for metastatic disease. Spine (Phila Pa 1976). 2004;29(19):2096-12.

14. Schinkel C, Gottwald M, Andress HJ. Surgical treatment of spondylodiscitis. Surg Infect (Larchmt). 2003:4(4):387-91.

15. Bilsky MH, Boland P, Lis E, Raizer JJ, Healey JH. Single-stage posterolateral transpedicle approach for spondylectomy, epidural decompression, and circumferential fusion of spinal metastases. Spine (Phila Pa 1976). 2000;25(17):2240-9.

16. Sasani M, Ozer AF. Single-stage posterior corpectomy and expandable cage placement for treatment of thoracic or lumbar burst fractures. Spine (Phila Pa 1976). 2009;34(1):E33-40. 